\title{
Research on vibration detection of GIS basin insulator
}

\author{
Ronghai Liu ${ }^{\mathrm{a}}$ \\ Yunnan Power Grid Electric Power Research Institute Co., Ltd., Kunming 650217, China
}

\begin{abstract}
This paper firstly introduces the importance of the GIS equipment basin insulator, and the serious impact of the basin type insulator damage. Later, the theoretical basis and method of vibration detection are introduced, and on the condition of intact and damaged of the basin insulator, the experiment of vibration detection were carried out. Later, the author analyzed the experimental data, and found that the vibration signal of the basin insulator in intact and damaged condition is different. It shows that it is feasible to detect whether the basin insulator is damaged by the vibration method, which provides a strong theoretical basis for the application of this method in engineering.
\end{abstract}

Keywords: GIS; basin insulator; vibration test.

\section{Introduction}

At present, most of the high voltage transmission lines used in the switch equipment is a combination of gas insulated switchgear equipment[1-3].Such equipment has covers an area of small, high reliability, long life cycle, low maintenance, device has the advantages of compact structure and easy to install. It can therefore be used in large power hub, in complex terrain or narrow terrain of the region are convenient in use. As the core component of GIS, the basin insulator will be used widely in the construction of UHV power grid, therefore, it will put forward higher requirements for its safe and stable operation.

In the GIS device with no stop level, the basin type insulator is one of the most important insulation parts, and its main function can be summarized as the following three aspects[4-6]:

(1)The center conductor and the GIS casing are isolated by the basin type insulator, and the high voltage bus bar of the different phases is also isolated, to keep the shell at the ground potential, to play the role of insulation, protection of the work safety, so the insulation level of the insulator is high enough.

(2)Two section high voltage bus conductor through the basin type insulator, they enter from one SF6 air chamber to another, the center conductor insert can be fixed high voltage conductors and plugin contact fixed bus.

(3)Gas diaphragm insulator has sealing effect on air chamber.GIS internal SF6 gas pressure, and once the leak will lead to a decline in the level of internal insulation. Therefore, the requirements of high air tightness, mechanical design requirements have a certain ability to withstand the pressure.

The above functions put forward higher requirements for the performance of the basin insulator,

\footnotetext{
a Corresponding author : 1343422033@qq. com
} 
and the mechanical strength of the basin insulator to achieve a certain tolerance. But in the process of operation, the inner stress of the insulator is likely to produce crack due to the mechanical extrusion of the center conductor and the outer shell.

According to the existing field operation failure and detection experience of GIS, it can be found that the early warning technology for the condition monitoring and defect of the basin type insulator is still not mature enough. When we can't effectively predict the existence of micro defects in advance, it is possible to lead to the further development of defects for serious fault accidents, so there is a big risk for the safe and stable operation of GIS.

In this paper, the method of vibration detection is put forward to detect the damage of GIS basin insulator. The research of the fault type vibration method for the crack defect of GIS basin insulator is studied, and an effective method to detect the crack defects of the basin insulator is proposed. This is of great significance to improve the accuracy and efficiency of the on-line monitoring of GIS basin type insulator, and ensure the normal and reliable operation of GIS equipment and ensure the safety and stability of the power grid.

\section{Vibration detection theory}

\subsection{Foundation of mechanical vibration}

Mechanical vibration system is a mechanical system that revolves around its static equilibrium position for reciprocating motion. The inertia, recovery and damping are the basic elements of the mechanical vibration system. Inertia is the nature of the current movement of the system, recovery is the nature of the system to restore the system to a state of equilibrium, damping is the nature of the energy consumption of the system. These three basic elements are usually characterized by mass $\mathrm{M}$, stiffness $\mathrm{K}$ and damping $\mathrm{C}$, as shown in figure 1.By these single degree of freedom system, a complex vibration system with multiple degrees of freedom can be formed. When there is a malfunction in the equipment, parts and components have defects, assembly or installation of changes in the situation, vibration amplitude, vibration type and spectral composition of equipment will be changed, and different defects and faults, the vibration caused by the way is also different [7-9].

When the equipment is in the abnormal vibration, the vibration measurement and analysis can reveal the existence and development of the equipment inside the hidden defects, which is conducive to timely repair to prevent the spread and development of the defect. When the abnormal vibration is not treated in time, it will make the equipment connection parts loose, material fatigue and lead to the damage $\Delta \omega_{i}^{2}=\frac{\phi_{i}^{T} \Delta K \phi_{i}}{\phi_{i}^{T} M \phi_{i}}$ of equipment.

An important physical parameter in mechanical vibration is the natural frequency, which is only related to the mass $\mathrm{m}$ of the spring constant $\mathrm{K}$ and the mass of the vibration system, and it has nothing to do with the initial conditions of motion.

Their relationships are shown in formula 1.

$$
\omega_{n}=\sqrt{\frac{k}{m}}
$$

\subsection{Vibration excitation}

Impact is a single pulse, its characteristics are: the process of sudden, short duration, great energy, usually it consists of zero to infinite harmonic components of all frequencies. The vibration frequency is basically in a certain range, and it has nothing to do with the frequency of the movement and the period of the external force; if the vibration damping is increased, the vibration speed will rise. 
Impulse hammer hammer excitation is a pulse hammer to knock to be measured system, to the system applied a impulse, and make it vibrate. It is a kind of fast and wide frequency excitation method, because the hammer force pulse has a flat spectrum curve in a certain frequency range.

Pulse hammer is a kind of exciting device which can generate transient exciting force, it consists of a hammer, hammer handle, and the weight composition. Equipped with a force sensor between the hammer and the hammer, this sensor can measure system by hammering force. Generally speaking, the size of the hammer force is determined by the movement speed of the system when the system is measured.

The frequency range of the force hammer excitation is mainly determined by the contact surface stiffness. The duration of the hammer material more hard pulse is shorter, and the maximum rate $f_{e}$ will be higher. In order to adjust the excitation frequency range, usually use a different material hammer.

When impulse excitation is used, it is equal to the force pulse of 1.5 sine wave applied to the system, as shown in figure 2 . The spectrum of this kind of pulse is in the frequency band less than the maximum frequency, and the spectrum of the pulse is essentially flat, and then decreased rapidly. In general, the duration of the hammer material more hard pulse is shorter, the higher the frequency limit.

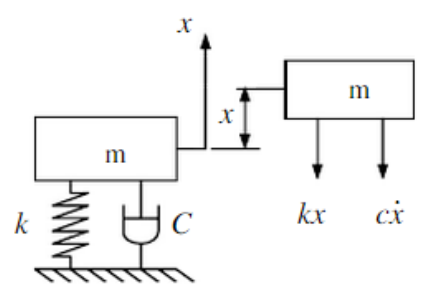

Figure 1. Single degree of freedom system model

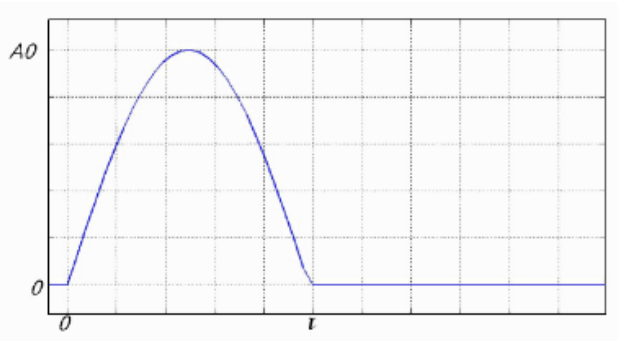

Figure 2. Pulse signal time domain waveform

\subsection{Vibration detection}

The eigenvalue problem of structural vibration is described by the following equation:

$$
\left(K-\omega^{2} M\right) \phi=0
$$

In this formula, $\mathrm{K}$ and $\mathrm{M}$ are the integral stiffness matrix and mass matrix, ${ }^{\phi}$ is a regular mode of vibration, $\omega$ is the inherent frequency. If the damage is caused by a small amount of the structural stiffness matrix and mass matrix, then the $\phi$ and ${ }^{\omega}$ have a small amount of change.The perturbation equation of the structural equation of motion is:

$$
\left\lfloor(K+\Delta K)-\left(\omega^{2}+\Delta \omega^{2}\right)(M+\Delta M)\right](\phi+\Delta \phi)=0
$$

In the formula, $\Delta K, \Delta M$ and $\Delta \phi$ are respectively the total stiffness matrix, mass matrix and the change of the mode of vibration.

For some complex structures, crack damage often has a more obvious effect on the structural stiffness, and almost no influence on the mass distribution.Such as the reduction of the stiffness of the basin insulator caused by the crack damage is much more significant than that of the mass, and in equation (3), $\Delta M$ can be regarded as equal to zero. When formula (3) is expanded and the two order term is ignored, then the formula (3) becomes: 


$$
\Delta \omega^{2}=\frac{\phi^{T} \Delta K \phi}{\phi^{T} M \phi}
$$

On a simple vibration mode $(\mathrm{i}=1,2, \ldots)$ :

$$
\Delta \omega_{i}^{2}=\frac{\phi_{i}^{T} \Delta K \phi_{i}}{\phi_{i}^{T} M \phi_{i}}
$$

It can be concluded that the variation of the natural frequency is a function of the change of the structure stiffness:

$$
\Delta \omega_{i}=f(\Delta K)
$$

According to formula (5) it is known that the change of structure stiffness $\mathrm{K}$ will cause the change of natural frequency $\omega_{i}$, and the structural stiffness is a direct impact on the mechanical strength of the structure, so the natural frequency of the structure will be able to reflect the changes in the structure of the damage.

We compare the damaged and the damaged insulator, and take the limit load as the reference point, The damage degree of the insulator can be expressed as the ratio between the limit load of the damaged insulator and the ultimate load of the intact insulator. The following relationships can be obtained:

$$
\frac{P_{1}}{P_{0}}=\frac{\omega_{i 1}^{2}}{\omega_{i 0}^{2}}
$$

In this formula: ${ }^{P_{0}}$ is the ultimate load of an insulator that is not damaged; ${ }^{P_{1}}$ is the limit load of the damaged insulator; ${ }^{\omega_{i 0}}$ is the natural frequency of the damaged insulator; ${ }^{\omega_{i 1}}$ is the natural vibration frequency of the damaged insulator;

$\mathrm{i}$ is the natural mode of vibration of insulators $(\mathrm{i}=1,2, \ldots)$. So it can solve the task of evaluating the mechanical state of the basin insulator by means of timely attention to the performance status of the natural frequency. We regard the basin insulator as a mechanical device with some degree of freedom, the summary of the mechanical vibration equation is written as follows:

$$
\frac{A d^{2} q}{d t^{2}}+\frac{B d q}{d t}+C q=F(t)
$$

In this formula: $A$ - inertia matrix elements; $B$ - dissipative force matrix; $C$ - quasi elastic coefficient matrix; $q$ - Summary coordinate matrix column; $F(t)$ - summary the force matrix.

\section{Experiment and data analysis}

\subsection{Laboratory equipment}

Acceleration sensor using the United States PCB company's general type three axis ICP accelerometer, parameters as shown in table 1.Hammer used PCB's 086C03 type hammer, hammer hammer neutral, parameters are shown in table 2 . The data acquisition was carried out using the modal test equipment 
Coco80 vibration inspection instrument, and the data were analyzed by using EDM software.

Table 1. Parameters of general three axis ICP accelerometer

\begin{tabular}{|c|c|c|c|c|c|}
\hline model & Range(g pk) & Sensitivity(mV/g) & $\begin{array}{c}\text { frequency } \\
\text { response(kHz) }\end{array}$ & $\begin{array}{c}\text { resolving } \\
\text { power(grms) }\end{array}$ & weight(gm) \\
\hline $\begin{array}{c}\text { 356A2 } \\
5\end{array}$ & \pm 200 & 25 & $1-5$ & 0.0002 & 10.5 \\
\hline
\end{tabular}

Table 2. The force hammer parameters of 086C03 model

\begin{tabular}{|c|c|}
\hline Name & $\begin{array}{c}\text { Impulse Hammer with force } \\
\text { sensor and tips }\end{array}$ \\
\hline range & $0-500 \mathrm{lbf} \mathrm{pk}$ \\
\hline sensitivity & $10 \mathrm{mV} / \mathrm{lb}( \pm 15 \%)$ \\
\hline Hammer & $2.5 \mathrm{kHz}$ \\
\hline resonant frequency & $\geq 22 \mathrm{kHz}$ \\
\hline Linear degree & $\leq 1 \%$ \\
\hline Excitation voltage & $18-30 \mathrm{VDC}$ \\
\hline Quality of force hammer & $0.16 \mathrm{~kg}$ \\
\hline Hammer diameter & $1.57 \mathrm{~cm}$ \\
\hline Hammer tip diameter & $0.63 \mathrm{~cm}$ \\
\hline Force hammer length & $21.6 \mathrm{~cm}$ \\
\hline
\end{tabular}

In the experiment, we first make a complete case of the model parameters of the basin insulator; Then, the basin type insulator is removed, and the modal experiment of a single insulator is carried out; Next, the basin type insulator is used to manufacture the crack, and the electric grinding wheel is used for the cutting process to form a crack, and then the modal experiment of the single insulator is carried out; Finally, the damaged insulator is re installed on the GIS interval device, and the modal parameters of the basin type insulator are measured.

The test is divided into 4 steps, respectively, the test of the basin type insulator in 4 different cases of the modal parameters of the case. The scientific and feasibility of detecting the damaged condition of the basin insulator by the vibration method is analyzed by comparing the results.

First step:

The modal experiment of the intact insulator installed on the GIS interval is carried out. In the selection of six acceleration sensor (1\# 6\#), followed by the distribution of distribution of $1 \# 2 \#$ sensors in the outer ring of the basin type insulator metal flange. $3 \# 4 \#$ sensors distribution in the basin type insulator on the right side of the metal flange parts. $5 \# 6 \#$ sensors distribution in basin type insulator on the left side of the flange part and on the left side of the cylindrical surface of the cavity. Its specific distribution as shown below.
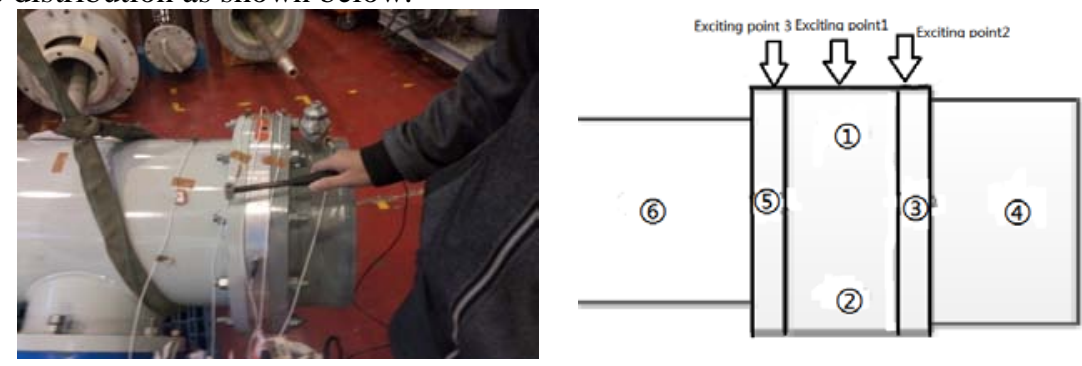

Figure 3. The distribution of the experimental excitation point and the pick-up point

The overall modal parameters of the basin insulator are studied by using the force hammer exciting point and 6 different position sensors. Through the modal experiment of the sample, the vibration signal of the vibration signal is detected, the 12 sets of data are generated when the excitation point is different and the location of the pick-up point is different. As shown in the figure below, when the 
excitation point in the basin of the outer ring flange of the outer flange of each sensor detected by the vibration signal spectrum.

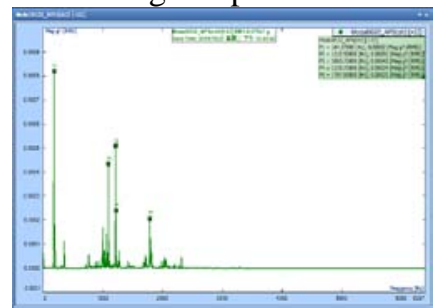

(a) excitation point 1 , sensor $1 \#$

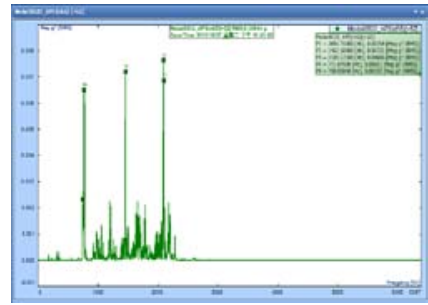

(d) excitation point 1 , sensor $4 \#$

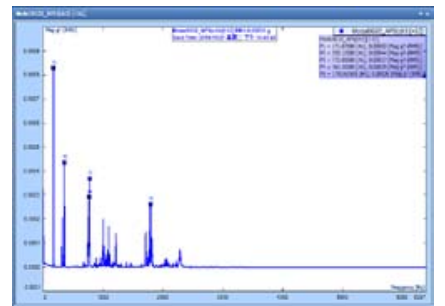

(b) excited point 1 , sensor $2 \#$

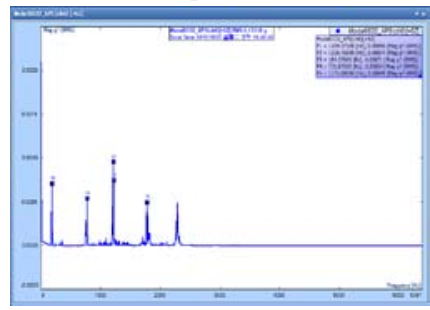

(e) excited point 1 , sensor $5 \#$

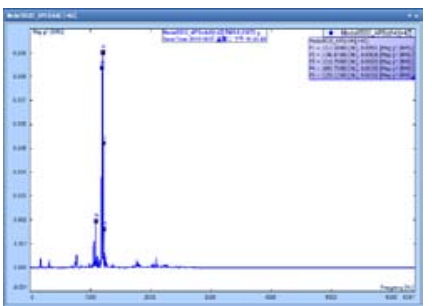

(c) exciting point 1 , sensor $3 \#$

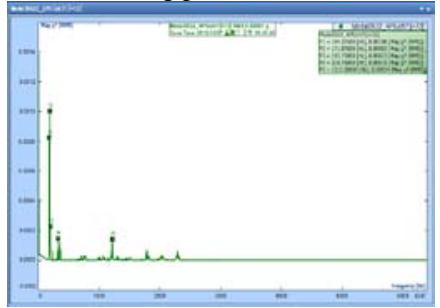

(f) exciting point 1 , sensor $6 \#$

Figure 4. The vibration signal spectrum of the excitation point on the flange of the outer ring flange of the basin type insulator

\section{Second step:}

Remove the basin type insulator in the first step, and carry out the modal experiment. According to the condition of the insulator structure, the 4 acceleration sensors are distributed on the sample. The force hammer excitation point is determined according to the location of the sensor. Sensors $2 \#$ and $1 \#$ are distributed on the outer flange ring surface of the insulator, which detect the vibration response of the flange ring. The sensor $3 \#$ is distributed on the convex surface of the insulator, which detects the vibration response of the insulator. The sensor $4 \#$ located in the center of hardware, its vibration response of hardware testing center. Hammer exciting point selection for outer ring flange is No. 1 , excitation point and resin insulator of the convex surface is No. 2 excitation point and center fittings is No. 3 exciting point, as shown in Figure 5.

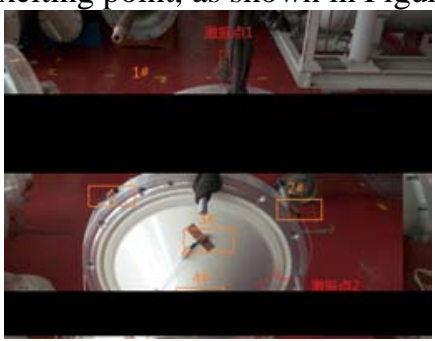

Figure 5. Experimental layout of single insulator

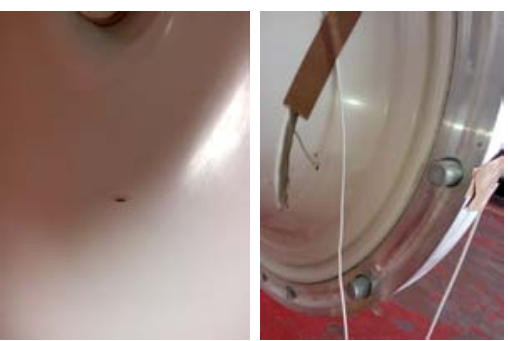

Figure 6. Damage of the basin insulator

Figure 7 shows the vibration spectrum of the signal measured by the sensor $1 \sim 4$, when the force hammer is exciting at the exciting point 1.

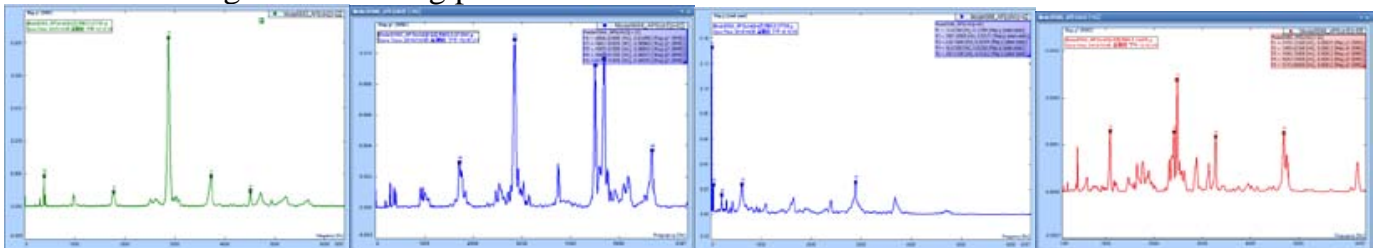

(a) excitation point 1 , sensor $1 \#$ (b) excited point 1, sensor 2\# (c) exciting point 1, sensor $3 \#$ (d) excitation point 1 , sensor $4 \#$

Figure 7. Vibrational spectrum 
Third step:

The basin type insulator is destroyed and cracks are formed,and the crack is a penetrating crack. The length of the crack profile on the convex surface is $12.2 \mathrm{~cm}$, width $0.88 \mathrm{~cm}$, the length of the crack profile on the concave surface is $1 \mathrm{~cm}$, the overall depth of the crack is $3.1 \mathrm{~cm}$, the specific case is shown in figure 6.

The distribution of the sensor and the position of the force hammer exciting is the same as that of the second step.Figure 8 shows the vibration spectrum of the signal measured by the sensor $1 \sim 4$, when the force hammer is exciting at the exciting point 1 .

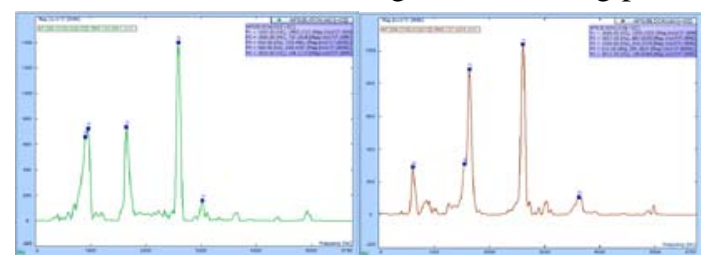

(a) excitation point 1 , sensor $1 \#$ (b) excited point 1 , sensor $2 \#$

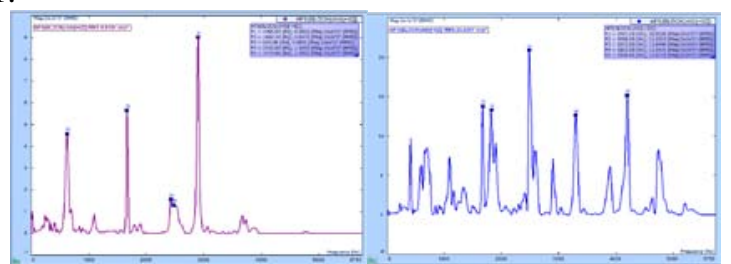

(c) exciting point 1 , sensor $3 \#$ (d) excitation point 1 , sensor $4 \#$

Figure 8. Vibrational spectrum

Fourth step:

The experiment was carried out on a single damaged insulator fitted on the GIS interval. The basin type insulator with a crack is re installed on the GIS interval, and the SF6 of each gas chamber is inflated to $0.4 \mathrm{MPa}$, and the modal experiment is carried out, the specific sensor distribution and the selection of the force hammer exciting point are consistent with the situation in the first step.Figure 9 shows the vibration spectrum of the signal measured by the sensor $1 \sim 4$, when the force hammer is exciting at the exciting point 1.

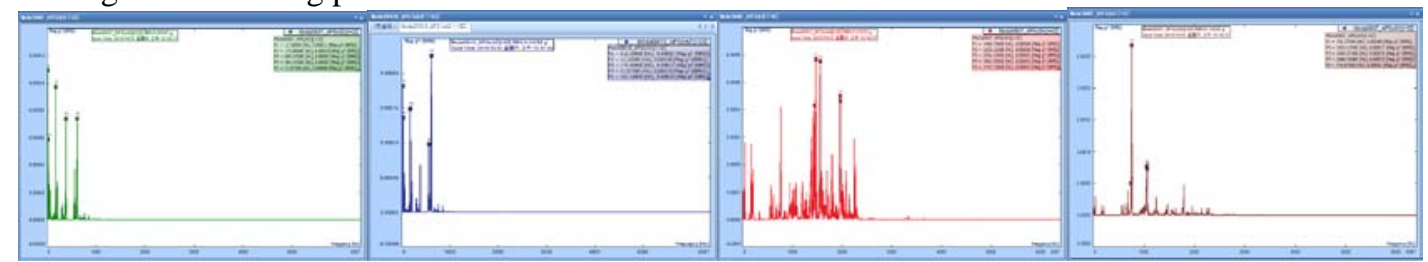

(a) excitation point 1 , sensor $1 \#(b)$ excited point 1 , sensor $2 \#$

(c) exciting point 1 , sensor $3 \#$ (d) excitation point 1 , sensor $4 \#$

Figure 9. Vibrational spectrum

\section{Data analysis and conclusion}

Conclusion can be found through the analysis of the experiment:

(1) When the basin type insulator is installed on the GIS interval, the existence or non existence of cracks in the basin insulator, the peak value of the vibration signal spectrum measured by the experimental personnel is very obvious, It can be seen from the spectrum, the low frequency part of the peak is more obvious, and it is easy to extract.

(2) For the same insulator, the characteristic frequency value of the vibration signal when it is not installed on the GIS is completely different from that of the vibration signal when it is installed on the GIS. A case study of a complete basin insulator: When it is not installed on the GIS, the frequency distribution of the vibration signal is more concentrated;when it is installed on the GIS, the frequency distribution of the vibration signal is more dispersed.

(3) When the damaged basin type insulator is not installed on the GIS, the maximum peak frequency is not stable, and the peak frequency points are mainly distributed in the $2000 \mathrm{~Hz}$, and the frequency distribution of the signal is relatively concentrated.

(4) The comparison of the two types of intact and damaged basin type insulator, when the insulator is in good condition, the frequency distribution of the vibration signal is relatively dispersed, and the amplitude of the range signal after $3500 \mathrm{~Hz}$ becomes zero; the frequency distribution of the vibration 
signal of the damaged insulator is more concentrated, and the signal amplitude at $2000 \mathrm{~Hz}$ is close to zero.

Above analysis to verify the feasibility of the vibration method of the basin insulator damage detection, it provides a theoretical basis for the detection of the basin insulator by vibration method.

\section{References}

1. Zhang Li. High voltage switchgear technology for gas insulated [D]. Nanchang University, (2014)

2. Shen Lu. Switchgear technology of $220 \mathrm{kV}$ gas insulated [J]. China electric power, 08:48-51, (2008).

3. Chen Kai, Wang Hui. Insulated switchgear busbar fault gas 220kV [J]. Power supply, 01:52-54 (2011).

4. Zhou Wenjun, Joe Shengya, Li Li, Wang Baoshan, Hu Hui, Luo Yunbai. The new features of CS_2 gas discharge along the surface of insulator in GIS [J]. High voltage technology, 03:848856, (2015).

5. Du Jinqiao, Li Naiyi, Peng Zongren, Zhang order, UHV AC insulator electric field distribution calculation and shield structure optimization [J]. High voltage technology, 12:3037-3043, (2013).

6. Li Bin. The necessity of the design of the metal outer ring and the inner ring of the GIS basin type insulator [J]. High voltage electrical equipment, 08:109-113, (2012).

7. Li Luping, Li Wangwang, Jin Fenghua, Li Haibo. Application of vibration detection technology in crack fault monitoring of wind turbine blades [J]. Thermal power engineering, 02:207212+222, (2013).

8. Yao Xin. Research on the detection technology of laser Doppler vibration [D]. Tianjin University, (2004)

9. Su Lili. The vibration detection technology in the application of vortex flowmeter [J]. Automation and instrumentation, 02:48-49, (2016). 